\title{
Mild Cognitive Impairment and Caregiver Burden: A Critical Review and Research Agenda
}

\author{
Perla Werner, $\mathrm{PhD}^{1}$
}

\begin{abstract}
Aging is a global phenomenon that is accompanied by an increase in the number of persons with non-communicable diseases including dementia. Since this development requires public health attention to meet the needs of the increasing numbers of elderly persons and their caregivers, scientific and clinical research in the area of dementia and cognitive disorders during the last decade has shifted to focus on the early diagnosis of the disease and, more specifically, on mild cognitive impairment (MCI). MCI is associated with severe consequences at the societal, individual and familial levels. However, compared to the wealth of studies in the area of caregiver burden and Alzheimer's disease, research in the area of MCI and caregiver burden is still developing. The aim of the present paper was to provide an analysis of the status of research regarding caregiver burden in MCI. This review indicated that despite important advances, gaps in the knowledge and understanding of caregiver burden in MCI remain. Only a clear delineation of the uniqueness of the concept of burden of care in MCI, accompanied by methodologically rigorous studies, will inform the development of interventions geared to reduce the burden of family members of persons with MCI.
\end{abstract}

Key words: Mild cognitive impairment, caregiver burden

Suggested Citation: Werner P. Mild cognitive impairment and caregiver burden: a critical review and research agenda. Public Health Reviews. 2012;34: epub ahead of print.

\section{INTRODUCTION}

Mild cognitive impairment (MCI) is a term used to refer to an intermediate medical state between normal aging and dementia. It is a condition characterized by newly acquired cognitive decline beyond what would be expected for an individual's age and education, yet leaving activities of

\footnotetext{
${ }^{1}$ Center for the Research and Study of Aging, University of Haifa, Israel.

Corresponding Author Contact Information: Professor Perla Werner at werner@ research. haifa.ac.il; Head, Center for the Research and Study of Aging, University of Haifa, Israel.
} 
daily living intact. ${ }^{1}$ Mild cognitive impairment has been given much scientific attention due to its potential significance in identifying dementia patients at an early stage (when decline in cognitive function has begun, but dementia has yet to ensue). However, the outcome of MCI is mixed, with some individuals remaining stable, deteriorating slowly, or even improving, while others deteriorate into a state of dementia. ${ }^{1-3}$ Studies assessing the course of cognitive deterioration over time show that the annual rate of conversion from MCI to dementia is ten percent to 15 percent, ${ }^{4}$ although it is estimated that between 20 percent and 50 percent of MCI patients remain stable or improve. ${ }^{5}$

Although there are substantial limitations in the ability to predict the transition from MCI to dementia or the rate of deterioration, ${ }^{3,5}$ it is clear today that $\mathrm{MCI}$ is associated with consequences at the societal, individual and familial levels. At the societal level, it has been estimated that the direct costs associated with MCI are now only slightly higher than the costs for persons without MCI, ${ }^{6}$ most probably because the topic has not been examined yet in detailed studies. However, it should be expected that as the knowledge of the syndrome advances, the costs will increase as a result of the development of new and unique services and interventions for those with the diagnosis and their family members. ${ }^{5,7}$

At the individual level, while still functioning in their day-to-day activities, persons with MCI are confronted with a variety of cognitive problems that cause them anxiety and distress. ${ }^{8-11}$ For example, several qualitative studies have shown that persons with MCI are confronted with feelings of social isolation, uncertainty and apprehension, which might be a result of the ambiguity of the diagnosis, of fear of embarrassment or from reduced self confidence. ${ }^{9,12}$

Finally, given these difficult psychological experiences, it is not surprising that the consequences of MCI at the family level are also detrimental. Indeed, there has been a marked increase in recent years in the number of studies assessing the effects of MCI on family caregivers. For example, a recent review covering the literature up to July 2011, indicated that the most commonly experienced emotions reported by caregivers of individuals with MCI were frustration, guilt, and anger. ${ }^{8}$ Other consequences associated with caring for a person with MCI are a strong sense of $\operatorname{loss}^{13}$ and deprivation in the caregiver relationship with the patient, ${ }^{14}$ including less effective communication, a loss of intimacy, ${ }^{8,15}$ and decreased marital satisfaction ${ }^{16}$ among spouses. Finally, the new responsibilities facing caregivers as a result of the need to compensate for the memory decline in those with MCI take a toll on the caregivers' own lifestyle, for example by 
reducing the amount of time they have to themselves ${ }^{17}$ or for participating in their own recreational activities. ${ }^{18}$

Caregiver burden is the main theoretical construct used in the literature to assess the negative consequences associated with caring for a person with dementia. The concept of caregiver burden has evolved from being viewed as a single construct to one encompassing multiple dimensions (e.g., subjective and objective components of burden) and multiple repercussions (e.g., negative and positive effects of caregiving) (see a review of caregiver burden in Etters, Goodall, and Harrison ${ }^{19}$ ).

The most prominent theory used to explain the causes of burden for family caregivers of persons with Alzheimer's disease (AD) is the Stress Process Model. ${ }^{20}$ In this model, the stress process includes three factors: sources, mediators or appraisals, and manifestations or outcomes. Sources of stress are divided into primary and secondary stressors. Primary stressors, which are derived directly from caregiving, include the cognitive and functional status of the person with $\mathrm{AD}$, the behavioral problems manifested, and the amount of care provided. ${ }^{21}$ Secondary stressors include strains arising from difficulties outside the caregiving situation, such as family conflict. The model hypothesizes that mediating factors, including appraisal and resources (both objective as well as social-psychological), can ameliorate the effect of the stressors on outcomes. Although a variety of mediators have been examined, ${ }^{22}$ the most common and well-documented mediators are caregiver burden and social support. ${ }^{21}$ The effects (outcomes) of this stress process on the caregivers are psychological distress (mainly depression), physical distress, and financial problems. ${ }^{23}$ Finally, the characteristics of the caregiver and the care recipient are conceptualized as context variables that influence all stages of the process.

Compared to the wealth of studies in the area of caregiver burden and $\mathrm{AD}$, research in the area of MCI and caregiver burden is still developing. The aim of the present paper is to provide an analysis of the status of research regarding caregiver burden in MCI. Although not a systematic review of the literature, this critical review was based on a computer-based literature search intended to identify publications on the topic of MCI and caregiver burden, published until May 2012. MEDLINE, AgeLine and PsycINFO databases were chosen for the search as they contain publications that cater to a wide range of health professions related to dementia care.

The following keyword search terms were used: MCI and burden. The search was restricted to publications in the English language on human subjects and was supplemented by manual searching of reference lists. Two researchers independently reviewed the titles and abstracts of the articles identified. 
In the present paper we will first provide background regarding MCI, followed by a discussion of the theoretical and methodological issues associated with caregiver burden in MCI. We will conclude by presenting implications for public health and by suggesting future research directions.

\section{DEFINITION AND EPIDEMIOLOGY}

The concept of MCI was introduced by the end of the previous century ${ }^{24}$ and since then has been recognized as a common condition in old age. However, considerable debate has emerged regarding its definition and assessment criteria., ${ }^{3,25,26}$ Moreover, there is substantial variety in the assessment of its incidence and prevalence. ${ }^{27}$ The present consensus states that MCI is characterized by clinical heterogeneity and that it includes amnestic (consisting of memory problems) and non-amnestic (consisting of impairment in other cognitive domains) phenotypes accompanied by subtypes of single and multiple domain classifications. ${ }^{28,29}$

Prevalence rates of MCI in population-based studies including participants aged 60 and over, range between three percent and 19 percent, with higher rates associated with increasing age and lower education, ${ }^{29-31}$ reaching even 42 percent among those aged 85 and above. ${ }^{32} \mathrm{~A}$ recent systematic review of nine studies assessing the incidence of MCI showed the incidence of amnestic MCI to range between 28 and 36 per 1,000 person-years and the incidence for any type of MCI to range between 51 and 77 per 1,000 person-years. ${ }^{33}$ An additional systematic review from 2012 found an incidence of 21.5 to 71.3 per 1,000 person-years for MCI (all subtypes), and 8.5 to 25.9 per 1,000 person-years for amnestic MCI. ${ }^{27}$

Despite the inconsistencies and variability in the epidemiological data regarding MCI, there is no doubt about the burden it imposes on those caring for persons with the syndrome.

\section{CAREGIVER BURDEN IN MCI}

Empirical studies assessing caregiver burden and MCI are summarized in Table 1. Their theoretical and methodological characteristics are discussed in the next sections. 


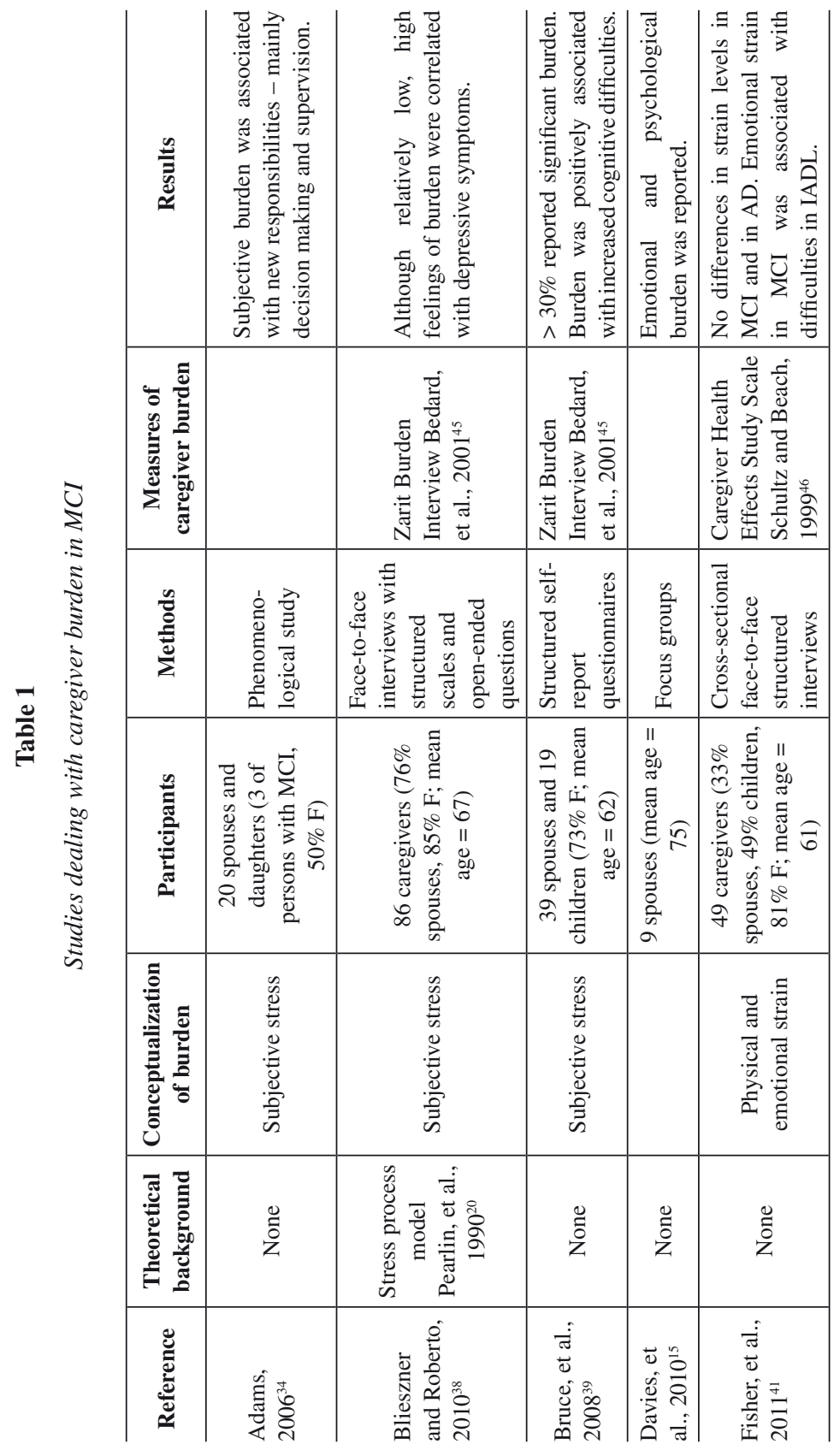




\begin{tabular}{|c|c|c|c|c|}
\hline 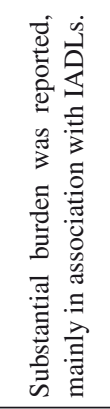 & 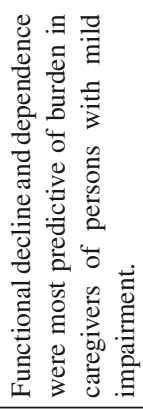 & 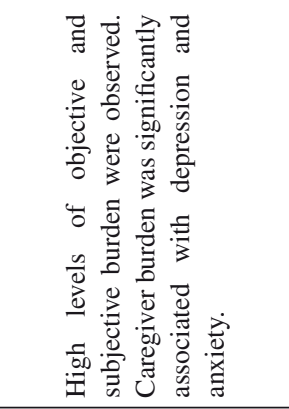 & 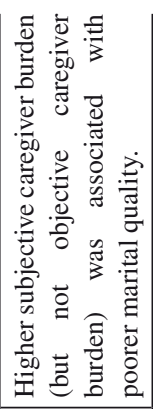 & 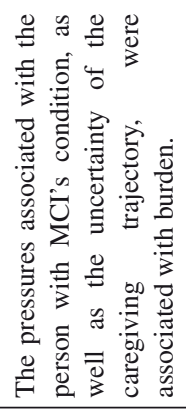 \\
\hline & 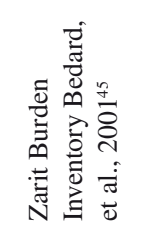 & 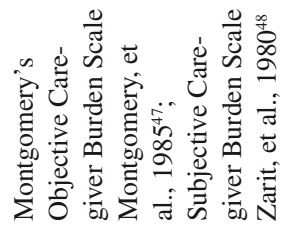 & 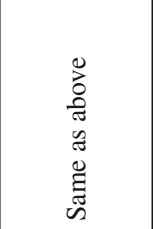 & \\
\hline 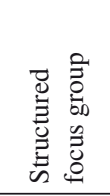 & 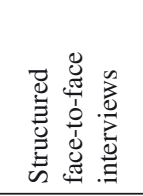 & 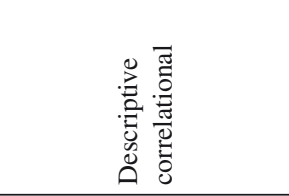 & 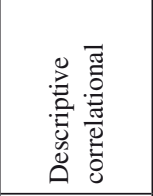 & 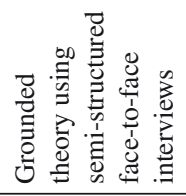 \\
\hline 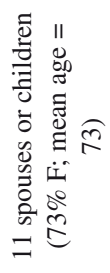 & 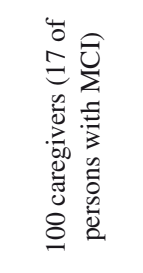 & 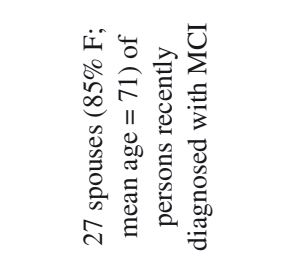 & 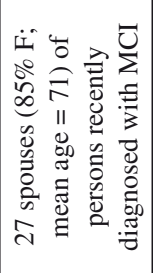 & 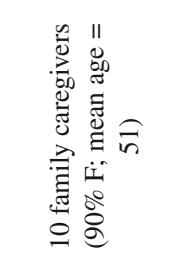 \\
\hline $\begin{array}{l}\text { बี } \\
0 \\
\vdots \\
\bar{\Xi} \\
\frac{0}{0} \\
0\end{array}$ & 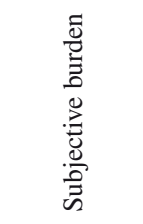 & 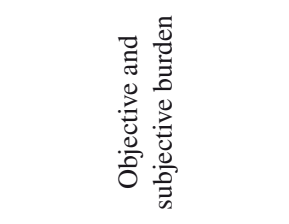 & 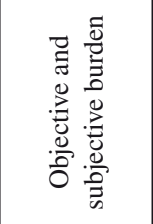 & \\
\hline 芩 & 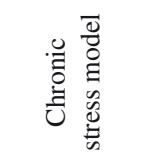 & 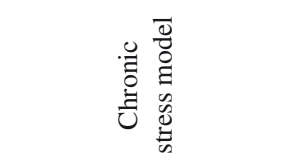 & 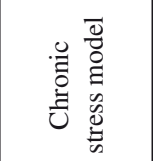 & \\
\hline 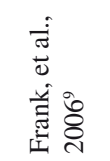 & 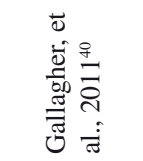 & 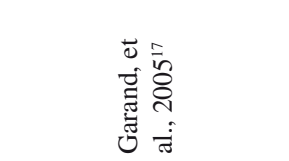 & 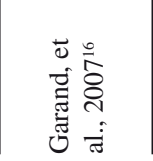 & 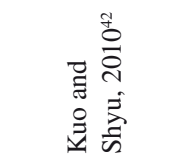 \\
\hline
\end{tabular}




\section{Theoretical Issues}

Overall, similar to the area of $\mathrm{AD}$, caregiver burden in MCI has been theoretically conceptualized as a multidimensional concept and has been framed mainly within the psychological perspectives of stress and coping.

Conceptualization of caregiver burden in MCI: The concept of caregiver burden has been generally conceptualized in the literature dealing with MCI as subjective stress, thus reflecting the extent to which the caregiver perceives the caregiving task to be a load on him/her, rather than its objective effects on health, daily chores and activities, and financial loss. This is surprising given that the assessment of objective burden is of utmost importance to the family caregiver, who often must take on new responsibilities in order to help the individual with MCI continue to function as long as possible. ${ }^{13}$ These responsibilities include housework, driving, financial management, supervision of daily activities, assistance in decision-making, and provision of psychological support, ${ }^{8,34}$ as well as some nursing tasks, such as administration of medications. ${ }^{17}$ However, only Garand and her colleagues ${ }^{16,17}$ assessed objective and subjective burden of care separately, showing that, similar to the area of $\mathrm{AD},{ }^{35}$ both aspects of burden are differentially associated with outcome variables, and that subjective burden (meaning the perceptions of the caregivers regarding their role) was more clearly associated with outcome variables such as depression and marital satisfaction.

Moreover, studies assessing the impact of burden in MCI have primarily concentrated on its negative impact, while ignoring its potential positive consequences. Studies in AD have shown that positive consequences, such as feeling responsible and finding meaning and fulfillment in caregiving, can considerably affect the outcome of the stress process, reducing ultimately its detrimental consequences. ${ }^{36,37}$ Thus, a wider conceptualization of caregiver burden and its impact in MCI may serve to extend our understanding in this area.

Theoretical framework: As in AD studies, the theoretical framework used in studies of caregiver burden in MCI has typically been the Stress Process Model..$^{20}$ However, it was empirically tested in its entirety only in the studies conducted by Blieszner and Roberto. ${ }^{38}$ By making use of a processoriented conceptual model to assess the complex interplay between the stressors and the internal and external coping factors that buffer the negative effects of the stressors, this approach enables a better understanding of the phenomenon. 
Correlates of caregiver burden in MCI: Overall, the main stressors affecting burden in MCI were similar to those found in studies assessing caregiver burden in AD. ${ }^{19}$ Indeed, a number of studies have shown that the burden of care in MCI is associated with the caregiver's perception of the patient's level of impairment and dependence. According to caregiver reports in one study, an association was identified between higher levels of caregiver burden and poorer cognitive functioning and more behavioral problems in the patient. ${ }^{39}$ Another study found that difficulties in performing Activities of Daily Living, Instrumental Activities of Daily Living (IADL) and perceived dependence were significantly associated with greater caregiver burden ${ }^{40}$ Similarly, a study on the caregivers of individuals with cognitive impairment no dementia (i.e., individuals whose cognitive functioning falls below normal but who do not meet dementia criteria) found that higher emotional strain among caregivers was associated with their reports of greater difficulty in performing IADL and more behavioral problems. ${ }^{41}$ Behavioral problems, though relatively low, were also found to be an important factor affecting caregiver burden in MCI. ${ }^{38}$ The similarity found in the correlates affecting caregiver burden in $\mathrm{AD}$ and $\mathrm{MCI}$ reflect not only the fact that MCI is conceptualized as a transitional stage between normal aging and $\mathrm{AD}$, but also the appropriateness of the theoretical assumptions underlying burden among persons with cognitive difficulties.

However, beyond those similarities, several studies reported on correlates that were unique to $\mathrm{MCI}$, such as watching a partner's memory decline $^{17}$ and dealing with clinginess. ${ }^{9}$ These findings are especially important in MCI as they might be associated with factors that could be changed in order to decrease the burden associated with caregiving for a person with MCI overtime.

Outcomes of caregiver burden: The majority of the studies in the area of MCI have shown that the burden and stress involved in taking care of a person with MCI was associated with negative repercussions on the mental health of the caregivers. In particular, high levels of anxiety and depression were reported in association with this stress..$^{14,17,38}$ Only in one study ${ }^{18}$ were detrimental effects on the physiological functioning of the caregiver for a person with $\mathrm{MCI}$ also reported.

\section{Methodological Issues}

Overall, studies examining caregiver burden in MCI are characterized by problems that are commonly found in new research fields, including sample, design, and assessment issues. 
Sample issues: The majority of the studies were based on small and nonrepresentative samples. Many researchers have relied on convenience samples of persons with MCI and caregivers attending community-based clinics. ${ }^{9,38-40,42}$ Three studies included participants who were recruited through registries for patients with AD. ${ }^{16,17,34}$ Only in the study by Fisher, et al. ${ }^{41}$ were the participants with MCI part of a nationally representative sample of respondents aged 70 and over.

Regarding cultural and ethnic characteristics, only two studies were conducted outside the United States, one in Ireland ${ }^{40}$ and the other in Taiwan. ${ }^{42}$ Almost all samples were made up primarily of female and Caucasian caregivers. Moreover, the use of small, non-representative samples has limited the ability to generalize and draw conclusions for populations other than those comprising the samples.

Design issues: Only four of the studies included in this review were qualitative, with two using focus group methodology $y^{15,42}$ and the other two based on phenomenological methodology. ${ }^{9,34}$ Blieszner and Roberto ${ }^{38}$ used mixed-method methodology. The lack of more qualitative research is surprising, since the study of caregiver burden in the area of MCI is relatively new and qualitative methods might provide a more thorough and dynamic understanding of the concept.

Regarding the quantitative studies reviewed, the majority were descriptive and cross-sectional. Indeed, only two provided an explicit hypothesis-testing process. ${ }^{38,39}$ The lack of prospective longitudinal studies limits our understanding of the dynamic development of caregiver's burden over time and over the course of cognitive deterioration. Blieszner and Roberto's study ${ }^{38}$ reported data from the first point in such a longitudinal study, and future follow-ups may provide us with additional insight.

Assessment issues: All quantitative studies included in the review used psychometrically validated tools to assess caregiver burden in the area of AD, mainly the full or short versions of the Zarit Burden Interview. The Zarit Burden Interview is a well validated instrument consisting of 22 items in its full version and 12 items in its short version assessing caregivers perceptions of their role in caregiving. ${ }^{17,38-40}$ Although the use of wellknown structured instruments generally reflects high methodological rigor, it should be noted that burden measures in the area of AD tend to focus on caregiving needs that emerge in more advanced stages of cognitive deterioration ${ }^{34}$ and less on difficulties arising in the early stages, such as decision-making and medication management problems. 


\section{LIMITATIONS OF THE REVIEW}

Although every attempt was made to include all the relevant literature, we can not disregard the possibility that some studies were not identified. In addition, information was synthesized and reported in summary tables, and no statistical techniques were used to assess methodological issues, such as sample size. However, it should be noted that this review was not intended to be an exhaustive review of the literature in the area, but rather to focus attention on its conceptual and methodological developments in order to provide guidelines for future research.

\section{CONCLUSIONS AND RESEARCH DIRECTIONS}

Dementia and cognitive disorders are among the major public health challenges of aging societies today. It has been estimated that approximately 36 million persons around the world are living with dementia, and this number will more than triple by the year $2050 .{ }^{43}$ As family members generally assume the role of primary caregivers ${ }^{44}$ at least as many family members are likely to be providing care or support for their relatives with dementia. It is not surprising therefore, that scientific and clinical research in the area of dementia during the last year has shifted to focus on the early diagnosis of the disease and, more specifically, on MCI, as an effort to prepare and prevent the consequences of dementia. Viewed as a predementia clinical stage bridging between normal aging and dementia, ${ }^{29}$ MCI poses considerable challenges not only for the individuals with MCI, but also for their family members. ${ }^{13}$ Indeed, we have witnessed a growing body of literature on the topic of caregiver burden in MCI. Expanding our knowledge on the strengths and pitfalls of these developments may allow us to more accurately estimate the societal costs along the entire spectrum of cognitive deterioration and to develop more effective interventions tailored to the specific needs of caregivers of persons at different stages of this spectrum.

Theoretically, studies assessing caregiver burden in MCI have adopted the same concepts and theoretical assumptions guiding the study of caregiver burden in AD. Accordingly, based on stress and coping theories, caregiver burden is also defined in the area of MCI as a multidimensional construct composed of two distinct components: subjective and objective burden. Likewise, it is described in terms of a complex interplay between the stressors and the internal and external coping factors that buffer the negative effects of the stressors. 
Adopting an existing theoretical framework has its advantage insofar as it provides a clear and coherent definition of the construct under study. However, the disadvantage in doing so lies in its emphasis on the aspects held in common between the burden of care in $\mathrm{MCI}$ and $\mathrm{AD}$, rather than on the unique characteristics and attributes of caregiver burden in MCI. Future studies should be encouraged to stress the unique rather than the similar factors affecting caregiver burden in the area of MCI. For example, such stressors as decision-making, degree of dependence, and management of medications should be added to the traditional stressors assessed by the Stress Process Model in AD. This is especially important as these early experiences are likely to influence and set the stage for many years of caregiving as cognitive deterioration advances. In addition, greater attention should be focused on the objective dimension of burden in MCI, as it might be more closely associated with the societal costs of care of MCI than the subjective dimension. Thus, future studies should concentrate on the assessment of the impact of caregiving for a person with MCI on the caregiver's use of time, difficulty with routine activities including employment duties and other social activities.

Any theoretical advancement in the understanding of the concept of caregiver burden in MCI must also be accompanied by improved methodological approaches. Our review of the existing literature stresses the need to move from a descriptive approach, based on small and non-representative samples, to large-scale studies based on representative samples, which will allow examination of the concept among different groups of caregivers, varying in gender, ethnicity, and relationship to the person with MCI. Additional qualitative or mixed-method methodology studies should also be promoted in order to facilitate a thorough understanding of the problem under study. Finally, longitudinal studies aimed at examining the changes in the significance of caregiver burden across time, in different cognitive and contextual stages, should be initiated.

In summary, this review indicates that despite important advances, gaps in the knowledge and understanding of caregiver burden in MCI remain. Only a clear delineation of the uniqueness of the concept of burden of care in MCI, accompanied by methodologically rigorous studies, will inform the development of interventions geared to reduce the burden of family members of persons with MCI. The implementation of the suggested strategies for coping with caregiver burden in MCI will require changes and increases in funding strategies in order to reduce the associated societal costs. 


\section{Acronyms List:}

$\mathrm{AD}=$ Alzheimer's disease

IADL $=$ Instrumental Activities of Daily Living

MCI = Mild cognitive impairment

Conflicts of Interest: None declared.

About the Author: Perla Werner, Ph.D. is Professor of Gerontology and Vice Rector in the University of Haifa. She is Head of the Center for Research and Study of Aging and her research examines psycho-social aspects of dementia and depression in elderly persons and their caregivers. She has published more than 200 manuscripts in peer-reviewed journals and received numerous competitive grants. She is currently conducting studies in the areas of stigma and Alzheimer's disease and its impact on persons with dementia, their caregivers and service providers.

\section{REFERENCES}

1. Gainotti G. Origins, controversies and recent developments of the MCI construct. Curr Alzheimer Res. 2010;7:271-9.

2. Olzaren J, Torrero P, Cruz I, Aparicio E, Sanz A, Mula N, et al. Mild cognitive impairment and dementia in primary care: the value of medical history. Fam Pract. 2011;28:385-92.

3. Werner P, Korczyn AD. Mild cognitive impairment: conceptual assessment, ethical, and social issues. Clin Interv Aging. 2008;3:413-20.

4. Frank AR, Petersen RC. Mild cognitive impairment. In: Handbook of clinical neurology. Duyckaerts C, Litvan I, (editors). Elsevier Health Sciences; 2008.

5. Pater C. Mild cognitive impairment (MCI) - the novel trend of targeting Alzheimer's disease in its early stages - methodological considerations. Curr Alzheimer Res. 2011;8:798-807.

6. Luppa M, Heinrich S, Matschinger H, Hensel A, Luck T, Riedel-Heller SG, Konig HH. Direct costs associated with mild cognitive impairment in primary care. Int J Geriatr Psychiatry. 2008;23:963-71.

7. Austrom M, Lu Y. Long term caregiving: helping families of persons with mild cognitive impairment cope. Curr Alzheimer Res. 2009;6:392-8.

8. Dean K, Wilcock G. Living with mild cognitive impairment: the patient's and carer's experience. Int Psychogeriatr. 2012;17:1-11.

9. Frank L, Lloyd A, Flynn JA, Kleinman L, Matza LS, Margolis MK, et al. Impact of cognitive impairment on mild dementia patients and mild cognitive impairment patients and their informants. Int Psychogeriatr. 2006; 18: 151-62.

10. Joosten-Weyn Banningh L, Vernooij-Dassen M, Olde Rikkert M, Teunisse JP. Mild cognitive impairment: coping with an uncertain label. Int J Geriatr Psychiatry.2008;23:148-54. 
11. Lingler JH, Nightingale MC, Erlen JA, Kane AL, Reynolds III, CF, Schulz R, DeKosky ST. Making sense of Mild Cognitive Impairment: a qualitative exploration of the patient's esperience. Gerontologist. 2006;46:791-800.

12. Brataas HV, Bjugan H, Wille T, Hellzen O. Experiences of day care and collaboration among people with mild dementia. J Clin Nurs. 2010;19:2839-48.

13. Lu YYF, Haase JE. Content validity and acceptability of the Daily Enhancement of Meaningful Activity Program: intervention for mild cognitive impairment patient-spouse dyads. J Neurosci Nurs. 2011;43:317-38.

14. Lu YF, Austrom MG, Perkins SM, Bakas T, Farlow MR, He F, et al. Depressed mood in informal caregivers of individuals with mild cognitive impairment. Am J Alzheimers Dis Other Demen. 2007;22:273-85.

15. Davies HD, Newkirk LA, Pitts CB, Coughlin CA, Sridhar SB, Zeiss L, Zeiss $\mathrm{AM}$. The impact of dementia and mild memory impairment (MMI) on intimacy and sexuality in spousal relationships. Int Psychogeriatr. 2010;22:618-28.

16. Garand L, Dew MA, Urda B, Hagerty Lingler J, DeKosky ST Reynolds III CF. Marital quality in the context of mild cognitive impairment. West J Nurs Res. 2007;29:976-92.

17. Garand L, Dew MA, Urda B, Lingler JH, Dekosky ST, Reynolds CF. Caregiving burden and psychiatric morbidity in spouses of persons with mild cognitive impairment. Int J Geriatr Psychiatry.2005; 20: 512-22.

18. Savla J, Roberto KA, Blieszner R, Cox M, Gwazdauskas F. Effects of daily stressors on the psychological and biological well-being of spouses of persons with mild cognitive impairment. J Gerontol B Psychol Sci Soc Sci. 2011;66:653-64.

19. Etters L, Goodall D, Harrison B. Caregiver burden among dementia patient caregivers: a review of the literature. J Am Acad Nurse Pract. 2008;20:423-8.

20. Pearlin LI, Mullan JT, Sample SJ, Skaff MM. Caregiving and the stress process: an overview of concepts and their measures. Gerontologist. 1990;30:583-94.

21. Pinquart M, Sörensen S. Ethnic differences in stressors, resources, and psychological outcomes of family caregiving: a meta-analysis. Gerontologist. 2005;45:90-106.

22. Campbell P, Wright J, Oyebode J, Job D, Crome P, Bentham P, et al. Determinants of burden in those who care for someone with dementia. Int J Geriatr Psychiatry. 2008;23:1078-85.

23. Carretero S, Garcés J, Ródenas F, Sanjosé V. The informal caregiver's burden of dependent people: theory and empirical review. Arch gerontol geriatr. 2009; 491:74-9.

24. Reisberg B, Ferris SH, de Leon MJ, Franssen ESE, Kluger, A, Mi P, et al. Stagespecific behavioral, cognitive, and in vivo changes in community residing subjects with age-associated memory impairment and primary degenerative dementia of the Alzheimer type. Drug Develop Resear. 1998;15:101-14. 
25. Ellison JM. A 60-year-old woman with mild memory impairment: review of mild cognitive impairment. JAMA. 2008;300:1566-74.

26. Portet F, Ousset PJ, Visser PJ, Frisoni GB, Nobili F, Scheltens P, et al. Mild cognitive impairment (MCI) in medical practice: a critical review of the concept and new diagnostic procedure. Report of the MCI Working Group of the European Consortium on Alzheimer's Disease. J Neurol Neurosurg Psychiatry. 2006;77:714-8.

27. Ward A, Arrighi HM, Michels S, Cedarbaum JM. Mild cognitive impairment: disparity of incidence and prevalence estimates. Alzheimer Dement. 2012; 8:14-21.

28. Neugroschl J, Wang S. Alzheimer's disease: diagnosis and treatment across the spectrum of disease severity. Mt Sinai J Med. 2011;78:596-612.

29. Petersen RC, Knopman DS, Boeve BF, Geda YE, Ivnik RJ, Smith GE, et al. Mild cognitive impairment: ten years later. Arch Neurol. 2009;66:1447-55.

30. Ganguli M, Chang CC, Snitz BE, Saxton JA, Vanderbilt J, Lee CW. Prevalence of mild cognitive impairment by multiple classifications: The MongongahelaYoughiogheny Healthy Aging Team (MYHAT) project. Am J Geriatr Psychiatry. 2010;18:674-83.

31. Petersen RC, Roberts RO, Knopman DS, Geda, YE, Cha RH, Pankratz VS, et al. Prevalence of mild cognitive impairment is higher in men: The Mayo Clinic Study of Aging. Neurology. 2010;75:889-97.

32. Yesavage J A, O'Hara R, Kraemer H, Taylor JL, Ferris S, Gely-Nargeot M, et al. Modeling the prevalence and incidence of Alzheimer's disease and mild cognitive impairment. J Psychiatr Res. 2002;36:281-6.

33. Luck T, Luppa M, Briel S, Riedel-Heller SG. Incidence of mild cognitive impairment: a systematic review. Dement Geriatr Cogn Disord. 2010;29: 164-75.

34. Adams KB. The transition to caregiving. J Gerontol Soc Work. 2006;47:3-29.

35. Wolfs CA, Kessels A, Severens JL, Brouwer W, de Vugt ME, Verhey FR, Dirksen CD. Predictive factors for the objective burden of informal care in people with dementia: a systematic review. Alzheimer Dis Assoc Disord. 2012;26:197-204.

36. Hilgeman MM, Allen RS, DeCoster J, Burgio LD. Positive aspects of caregiving as a moderator of treatment outcome over 12 months. Psychol Aging. 2007;22:361-71.

37. McLennon SM, Habermann B, Rice M. Finding meaning as a mediator of burden on the health of caregivers of spouses with dementia. Aging Ment Health. 2011;15:522-30.

38. Blieszner R, Roberto KA. Care partner responses to the onset of mild cognitive impairment. Gerontologist. 2010;50:11-22.

39. Bruce JM, McQuiggan M, Williams V, Westervelt H, Tremont G. Burden among spousal and child caregivers of patients with mild cognitive impairment. Dement Geriatr Cogn Disord. 2008;25:385-90. 
40. Gallagher D, Mhaolain AN, Crosby L, Ryan D, Lacey L, Coen RF, et al. Dependence and caregiver burden in Alzheimer's disease and mild cognitive impairment. Am J Alzheimers Dis Other Demen. 2011;26:110-4.

41. Fisher GG, Franks MM, Plassman BL, Brown SL, Potter GG, Lleweylln D, et al. Caring for individuals with dementia and cognitive impairment, not dementia: findings from the Aging, Demographics, and Memory Study. J Am Geriatr Soc. 2011;59:488-94.

42. Kuo LM, Shyu YIL. Process of ambivalent normalisation: experience of family caregivers of elders with mild cognitive impairment in Taiwan. J Clin Nurs. 2010;19:3477- 84 .

43. World Health Organization. Dementia: a public health priority. UK: WHO Library; 2012.

44. Martin Y, Gilbert P, Mcewan K, Irons C. The relation of entrapment, shame and guilt to depression in carers of people with dementia. Aging Mental Health. 2006;10,101-6.

45. Bedard M, Molloy DW, Squire L, Dubois S, Lever JA, O'Donnell M. The Zarit Burden Interview: a new short version and screening version. Gerontologist. 2001;41:652 -7.

46. Schulz R, Beach SR. Caregiving as a risk factor for mortality: The Caregiver Health Effects study. JAMA. 1999;282:2215-9.

47. Montgomery RJV, Gonyea JG, Hooyman NR. Caregiving and the experience of subjective and objective burden. Fam Relations. 1985;34:19-26.

48. Zarit SH, Reever KE, Bach-Peterson J. Relatives of the impaired elderly: correlates of feelings of burden. Gerontologist. 1980;20,649-55. 